\title{
Anti-exudation effects of sodium ferulate and oxymatrine combination via modulation of aquaporin 1
}

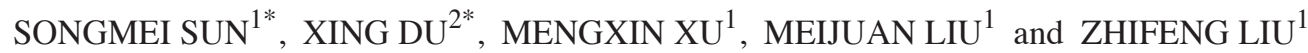 \\ ${ }^{1}$ Basic Medical Department, School of Pharmacy, Yantai University, Yantai, Shandong 264005; \\ ${ }^{2}$ Pharmacy Department, Yantai Yuhuangding Hospital, Yantai, Shandong 264000, P.R. China
}

Received July 21, 2015; Accepted April 21, 2017

DOI: $10.3892 /$ etm.2017.4679

\begin{abstract}
The present study aimed to investigate the anti-exudative effects of sodium ferulate combined with oxymatrine in a mouse model of acetic acid-induced peritonitis. Furthermore, the underlying mechanisms were explored by determining the effects of these drugs on the volume and aquaporin 1 (AQP1) expression in vascular endothelial cells on omentum majus and human umbilical vein endothelial cells (HUVEC). Treatment with sodium ferulate combined with oxymatrine was shown to significantly inhibit acetic acid-induced vascular permeability in the peritonitis model mice and furthermore to significantly decrease the optical density of Evans blue, the leukocyte number and the levels of interleukin-6, C-reactive protein and interferon- $\gamma$ in peritoneal lavage fluid. Pathological analysis of the omentum majus revealed that sodium ferulate and oxymatrine combination treatment significantly alleviated vascular endothelial cell edema and capillary loss. In vitro, flow cytometry revealed that the volume of HUVECs was significantly reduced in the drug treatment groups, as reflected in the forward scatter value. The optical density of AQP1 on the membrane of the vascular endothelial cells on omentum majus and HUVECs were significantly increased in the drug treatment groups compared with the model group. These results indicated that sodium ferulate and oxymatrine combination treatment possessed prominent anti-exudative effects and that the underlying mechanisms are likely to include the improvement of vascular endothelial cellular edema, possibly by upregulation of AQP1 expression on their membrane, which requires further exploration.
\end{abstract}

Correspondence to: Professor Zhifeng Liu, Basic Medical Department, School of Pharmacy, Yantai University, 30 Qingquan Road, Laishan, Yantai, Shandong 264005, P.R. China

E-mail: liuzhifengytu@163.com

*Contributed equally

Key words: sodium ferulate, oxymatrine, inflammation, anti-exudation, peritonitis, aquaporin 1

\section{Introduction}

Sodium ferulate extracted from Angelica sinensis and Oxymatrine, an alkaloid extracted from Sophorae flavescentis, have been reported to possess anti-inflammatory and anti-oxidant effects $(1,2)$. Previous studies revealed that a combination of sodium ferulate and oxymatrine exerted synergistic anti-inflammatory effects (3-6). The studies demonstrated that sodium ferulate and oxymatrine combination significantly inhibited xylene-induced edema in mouse ears and carrageenan-induced edema in rat paws. A mechanistic study revealed that the anti-inflammatory effects of sodium ferulate and oxymatrine combination was mainly associated with its modulatory effect on interleukin-6 (IL-6), IL-11, C-reactive protein (CRP) and interferon- $\gamma($ IFN- $\gamma)$ in a RAW 264.7 cell model stimulated by lipopolysaccharides (4), which had also been verified in mouse models of cecal ligation and puncture-induced sepsis and enthanol-induced liver damage as well as in a RAW 264.7 cell model of lipopolysaccharide-stimulated damage $(5,6)$.

Inflammation is a defensive response of the body to pathogenic agents, which is a common and important basic pathological process in the development of diseases $(7,8)$. An appropriate inflammatory response is beneficial for the body to eliminate the invading pathogens, but an exaggerated inflammatory response, such as that to lung edema, peritonitis and sepsis, is harmful for the body (9). Therefore, anti-inflammatory drugs are widely used in the clinic. Study of the inhibition of cyclooxygenase activity is an important route for the screening of non-steroidal anti-inflammatory drugs. However, preliminary experiments by our group found that the combination of sodium ferulate and oxymatrine did not exhibit any synergistic inhibitory effect on cyclooxygenase (data not shown). The present study further explored the anti-inflammatory effects of this drug combination and focused on the underlying mechanism.

Aquaporins (AQPs) are cell-membrane proteins that have been reported to have a vital role in transporting water across cell membranes (10). The present study hypothesized that sodium ferulate and oxymatrine may exert their anti-inflammatory exudation effect to reduce vascular endothelial cellular edema by affecting AQPs. In the present study, a mouse model of acetic acid-induced peritonitis was used to evaluate the effects of sodium ferulate and oxymatrine on anti-inflammatory exudation. By observation of the cell volume and AQP1 
expression by omentum majus vascular endothelial cells and human umbilical vein endothelial cells (HUVECs), an approach was made to explain the anti-inflammatory exudation mechanism.

\section{Materials and methods}

Materials. Sodium ferulate [molecular formula, $\mathrm{C}_{10} \mathrm{H}_{9} \mathrm{NaO}_{4}$ x 2 $\mathrm{H}_{2} \mathrm{O}$; molecular weight, 252.20; Chemical Abstracts Service (CAS) no. 24276-84-4; high-performance liquid chromatography (HPLC) purity, >99\%], oxymatrine (molecular formula, $\mathrm{C}_{15} \mathrm{H}_{24} \mathrm{~N}_{2} \mathrm{O}_{2} \times \mathrm{H}_{2} \mathrm{O}$; molecular weight, 282.38; CAS no. 16837-52-8; HPLC purity, >98\%) were provided by Beijing SL Pharmaceutical Co. Ltd. (Beijing, China). Sodium ferulate and oxymatrine were combined at a molar ratio of 1:2, which was determined by preliminary pharmaceutical and pharmacological tests (data not shown). At this molar ratio, the solution system was most stable with a $\mathrm{pH}$ value of 7.0, and the pharmacological activity was also the best in vivo and in vitro, (unpublished data). The primary AQP1 antibody was purchased from Santa Cruz Biotechnology, Inc. (cat. no. SC-20810; Dallas, TX, USA). The horseradish peroxidase-conjugated secondary antibody was obtained from Zhongshan Golden Bridge Biotechnology Co. Ltd. (cat. no. SPN-9001; Beijing, China). ELISA kits for measurement of the levels of IL-6, CRP and IFN- $\gamma$ were purchased from Groundwork Biotechnology Diagnosticate Ltd. (San Diego, CA, USA). Evans blue was purchased from Sinopharm Chemical Reagent Co., Ltd. (Shanghai, China). Dexamethasone was purchased from Shandong Lukang Pharmaceutical Co., Ltd. (Jining, China). Bovine serum albumin was obtained from Bai Qian Biotechnology Co., Ltd. (Shanghai, China). Diaminobenzidine was purchased from Zhongshan Golden Bridge Biotechnology Co.,Ltd. RPMI-1640 medium, fetal bovine serume and trypsin were produced by Gibco; Thermo Fisher Scientific, Inc. (Waltham, MA, USA). HUVECs were purchased from the Insitute of Biochemistry and Cell Biology (Shanghai Institutes for Biological Sciences, Shanghai, China).

Animals. Male Swiss mice (400 mice, at 4 weeks of age; Shandong Luye Pharmaceutical Co. Ltd., Yantai, China; quality certificate no. Lu 20090009) were used. The animals were kept under standard conditions (12-h light/dark cycle; temperature, $23 \pm 2^{\circ} \mathrm{C}$; humidity, $55 \pm 5 \%$ ) and adapted to the surrounding environment for 3 days prior to the experiments. In order to reduce the animal individual differences, the healthy animals, weighting 18-22 $\mathrm{g}$ were selected for use in the subsequent experiments, because the drugs were administered on the basis of the animal weight $(\mathrm{mg} / \mathrm{kg})$. Other animals were returned to the animal department. The animals fasted, with access to water only for $12 \mathrm{~h}$ prior to the experiment, and following administration of drugs to the animals, food and water were provided ad libitum. The Experimental Animal Management Center of Yantai University (Yantai, China) approved all animal procedures of this study in accordance with the NIH Guidelines (11) for the Care and Use of Laboratory Animals.

Exudation inhibition ratio of Evans blue in mice with acetic acid-induced peritonitis. In order to investigate the anti-exudation activity of sodium ferulate and oxymatrine combination, the mice were injected with Evans blue and peritonitis was induced with acetic acid according to a previously described method (12). Mice used in the experiment were randomly divided into a normal control group (saline), a model group (saline and acetic acid), 5 sodium ferulate groups $(15.6,31.3,62.5,125$ and $250 \mathrm{mg} / \mathrm{kg}), 5$ oxymatrine groups $(15.6,31.3,62.5,125$ and $250 \mathrm{mg} / \mathrm{kg}$ ) and 5 sodium ferulate + oxymatrine combination groups $(4.8+10.8,9.7+21.6$, $19.4+43.1,38.8+86.2$ and $77.5+172.5 \mathrm{mg} / \mathrm{kg}$, respectively). Each group contained 10 animals. The animals were intraperitoneally injected with the corresponding drug or drug combination in saline at a volume of $10 \mathrm{ml} / \mathrm{kg}$. One hour later, $0.5 \%$ Evans blue solution $(0.2 \mathrm{ml} /$ mouse $)$ was intravenously injected, followed by intraperitoneal injection of $0.9 \%$ acetic acid solution $(0.2 \mathrm{ml} / \mathrm{mouse})$. After $20 \mathrm{~min}$, the animals were sacrificed and the peritoneal cavity was washed with $1 \mathrm{ml}$ saline. Subsequently, the peritoneal lavage fluid was collected and centrifuged at $1,000 \mathrm{x}$ for $15 \mathrm{~min}$ at $4^{\circ} \mathrm{C}$. The supernatant was isolated and the optical density (OD) value of Evans blue was measured at $590 \mathrm{~nm}$. The inhibition ratio of Evans blue was calculated using following formula: Exudation inhibition ratio $=\left(1-\mathrm{OD}_{2} / \mathrm{OD}_{1}\right) \times 100 \%$, with $\mathrm{OD}_{1}$ being the optical density of Evans blue in the peritoneal lavage fluid of the model group and $\mathrm{OD}_{2}$ being the optical density of Evans blue in the peritoneal lavage fluid of the respective drug treatment group.

Number of leukocytes and the levels of IL-6, CRP and IFN- $\gamma$ in peritoneal lavage fluid. The animals were randomly divided into a normal control group (saline), a model group (saline and acetic acid), dexamethasone group (DEX, $1 \mathrm{mg} / \mathrm{kg}$ ), sodium ferulate group $(19.4 \mathrm{mg} / \mathrm{kg})$, oxymatrine group $(43.1 \mathrm{mg} / \mathrm{kg})$ and 3 sodium ferulate + oxymatrine combination groups $(9.7+21.6$, $19.4+43.1$ and $38.8+86.2 \mathrm{mg} / \mathrm{kg}$ ). Each group contained 10 animals. One hour after intraperitoneal injection with the corresponding drug or drug combination in saline $(10 \mathrm{ml} / \mathrm{kg})$, $0.9 \%$ acetic acid solution $(0.2 \mathrm{ml} /$ mouse $)$ was intraperitoneally injected. After $6 \mathrm{~h}$, the animals were sacrificed and the peritoneal cavity was washed with $1 \mathrm{ml}$ saline. The peritoneal lavage fluid was collected and centrifuged at 1,000 x $\mathrm{g}$ for $15 \mathrm{~min}$ at $4^{\circ} \mathrm{C}$. The supernatant and sediment were isolated for the measurement of IL-6, CRP and INF- $\gamma$ by ELISA in accordance with the manufacturer's instructions, and analysis of the number of leukocytes by a hematocyte counter, respectively.

Pathological and immunohistological analysis of omentum majus tissue. The animals were randomly divided into a normal control group (saline), a model group (saline and acetic acid), sodium ferulate group (19.4 mg/kg), oxymatrine group $(43.1 \mathrm{mg} / \mathrm{kg})$ and 3 sodium ferulate and oxymatrine combination groups $(9.7+21.6,19.4+43.1$ and $38.8+86.2 \mathrm{mg} / \mathrm{kg})$. Each group contained 10 animals. Treatment with drugs and acetic acid was performed as above. At $20 \mathrm{~min}$ after the last injection, the animals were sacrificed.

The omentum majus tissues were rapidly isolated and fixed with $10 \%$ formalin for $24 \mathrm{~h}$ for pathological analysis. The specimens were cut into $4-\mu \mathrm{m}$ sections and stained with hematoxylin and eosin. The damage degree of omentum majus was evaluated by an independent observer (Pathological 
Department, Shandong Luye Drug Safety Evaluation Center Yantai, China; Good Laboratory Practice Lab as certified by the Chinese State Food and Drug Administration), who was blinded to the grouping. The damage score were obtained on the basis of vascular endothelial cellular morphology and blood capillary morphology. The scoring criteria for the surface morphology of vascular endothelial cells were as follows: 0, no significant change of cell surface morphology; 1 , large number of villi and few cellular edema; 2 , loss of presudopodium and edema of various cells; 3 , significant protrusion into the lumen and formation of a cell gap (13). The scoring criteria of damaged capillaries were as follows: 0 , integrated capillaries with no significant damage; 1 , mild damage but hardly any shedding of capillaries; 2 , moderate damage with a small amount of capillaries shedding; 3 , a large number of capillaries shedding (14).

For immunohistochemical analysis, omentum majus tissue samples were washed with physiological saline and fixed in $4 \%$ paraformaldehyde for $24 \mathrm{~h}$. The specimens were then dehydrated and embedded in paraffin. The tissues were cut into 4- $\mu \mathrm{m}$ sections, deparaffinized and hydrated in PBS ( $\mathrm{pH} 7.4$ ). The sections were treated with $3 \% \mathrm{H}_{2} \mathrm{O}_{2}$ at room temperature for 15 min to block endogenous peroxidase activity and blocked with $1 \%$ bovine serum albumin (BSA) for $20 \mathrm{~min}$. The sections were sequentially incubated overnight at $4^{\circ} \mathrm{C}$ with goat polyclonal antibody against AQP1 (dilution, 1:200 in PBS). The sections were then washed with PBS and incubated with horseradish peroxidase-conjugated secondary antibody (1:100) at room temperature for $15 \mathrm{~min}$. After washing, the sections were incubated with diaminobenzidine for $3 \mathrm{~min}$ at room temperature. Finally, sections were counterstained with Mayer's hematoxylin. In the negative control, staining was performed using $0.01 \mathrm{M}$ PBS instead of the primary antibody. Following initial examination of hematoxylin and eosin-stained slides, the most appropriate sections were selected for semi-quantitative immunohistochemical analysis. AQP1 expression was determined using a semi-quantitative immunohistochemical method (15).

Cell culture. HUVECs were maintained in RPMI-1640 medium supplemented with $10 \%(\mathrm{v} / \mathrm{v})$ fetal bovine serum, $100 \mathrm{U} / \mathrm{ml}$ of penicillin and $100 \mu \mathrm{g} / \mathrm{ml}$ streptomycin. Cells were incubated at $37^{\circ} \mathrm{C}$ in a humidified atmosphere containing $5 \% \mathrm{CO}_{2}$. The medium was routinely changed every two days.

Assessment of the volume change of HUVECs. HUVECs were seeded in a 6 -well plate at a density of $1 \times 10^{4}$ cells $/ \mathrm{ml}$ in $3 \mathrm{ml}$ and incubated for $24 \mathrm{~h}$. The cells were treated with sodium ferulate $(100 \mu \mathrm{mol} / \mathrm{l})$ or oxymatrine $(200 \mu \mathrm{mol} / \mathrm{l})$, or sodium ferulate + oxymatrine combination $(50+100,100+200$ or $200+400 \mu \mathrm{mol} / \mathrm{l})$. After incubation for $1 \mathrm{~h}$, the cells were stimulated with acetic acid $(8 \mathrm{mmol} / \mathrm{l})$, except for the cells in the control well. After incubation for $20 \mathrm{~min}$, the cells were diluted with PBS and collected by trypsinization. In a suspension with a density of $1 \times 10^{9}$ cells/l, the volume of the cells was detected by flow cytometry (BD FACS AriaII; BD Biosciences, San Jose, CA, USA).

Immunocytochemical staining and microscopic examination. HUVECs were seeded onto coverslips in a 24-well plate (1x10 $0^{5}$ per well) and incubated in complete medium, containing fresh RPMI-1640 medium supplemented with $10 \%$ (v/v) fetal bovine serum at $37^{\circ} \mathrm{C}$ under a $5 \% \mathrm{CO}_{2}$ for $24 \mathrm{~h}$. The cells were treated with different concentrations of sodium ferulate $(25$, $50,100,200$ or $400 \mu \mathrm{mol} / \mathrm{l}$ in medium) or oxymatrine $(50,100$, 200,400 or $800 \mu \mathrm{mol} / 1$ in medium), or sodium ferulate and oxymatrine combination $(25+50,50+100,100+200,200+400$ or $400+800 \mu \mathrm{mol} / 1$ in medium), and incubated for $1 \mathrm{~h}$. Acetic acid was then added to the corresponding wells at a concentration of $8 \mathrm{mmol} / \mathrm{l}$. The same amount of solvent was added to the control wells. After $20 \mathrm{~min}$ of incubation, the cells were washed with PBS.

For immunocytochemical staining, the cells were fixed with $4 \%$ paraformaldehyde for $15 \mathrm{~min}$ and dried in air for 5 min (16). Subsequently, cells on coverslips were blocked using $3 \% \mathrm{H}_{2} \mathrm{O}_{2}$ for $15 \mathrm{~min}$ at room temperature and rinsed with PBS as described previously (17). After blocking, cells on coverslips were blocked with $1 \%$ BSA in PBS for 15 min at $37^{\circ} \mathrm{C}$. Next, the cells on coverslips were incubated with goat polyclonal AQP1 antibody $(1: 200)$ overnight at $4^{\circ} \mathrm{C}$. After rinsing with $\mathrm{PBS}$, the horseradish peroxidase-conjugated secondary antibody (1:100) were used at room temperature for 15 min (18). Following a further wash with PBS, the cells on coverslips were incubated with diaminobenzidine for $3 \mathrm{~min}$ at room temperature. Finally, samples were washed and stained with Mayer's hematoxylin. Coverslips were mounted on microscope slides. Semi-quantitative assessment was performed to evaluate the expression of AQP1 $(19,20)$. The optical density was determined with Image-pro plus version 6.0 software (Media Cybernetics, Inc., Rockville, MD, USA).

Statistical analysis. Values are expressed as the mean \pm standard deviation. Differences among groups were analyzed using one-way analysis of variance followed by the unpaired Student's t-test with equal variance. SPSS 17.0 statistical software (SPSS, Inc., Chicago, IL, USA) was used for statistical analysis. $\mathrm{P}<0.05$ was considered to indicate a statistically significant difference.

\section{Results}

Effects of sodium ferulate and oxymatrine administered alone or in combination on exudation of Evans blue in mice with acetic acid-induced peritonitis. The anti-exudation effects of the drugs were assessed in a mouse model of acetic acid-induced peritonitis. The results demonstrated that treatment with sodium ferulate or oxymatrine alone inhibited Evans blue leakage when the dose was $>62.5 \mathrm{mg} / \mathrm{kg}$ (Fig. 1A and B). The highest inhibition rate was $37.71 \%$ for sodium ferulate and $41.85 \%$ for oxymatrine. The concentration leading to $50 \%$ inhibition ( ID $_{50}$ value) was $372.0 \mathrm{mg} / \mathrm{kg}$ for sodium ferulate and $310.3 \mathrm{mg} / \mathrm{kg}$ for oxymatrine. However, treatment with sodium ferulate and oxymatrine combination at $>9.7+21.6 \mathrm{mg} / \mathrm{kg}$ significantly increased the exudation inhibition ratio of Evans blue in an obvious dose-dependent manner (Fig. 1C). The highest inhibition rate was $59.74 \%$. The $\mathrm{ID}_{50}$ value was $46.5+104.1(\mathrm{mg} / \mathrm{kg})$, which was far less than that of sodium ferulate or oxymatrine alone. The isobologram analysis showed that the $\mathrm{ID}_{50}$ value of sodium ferulate and oxymatrine combination was below the isobol of the theoretical additive 

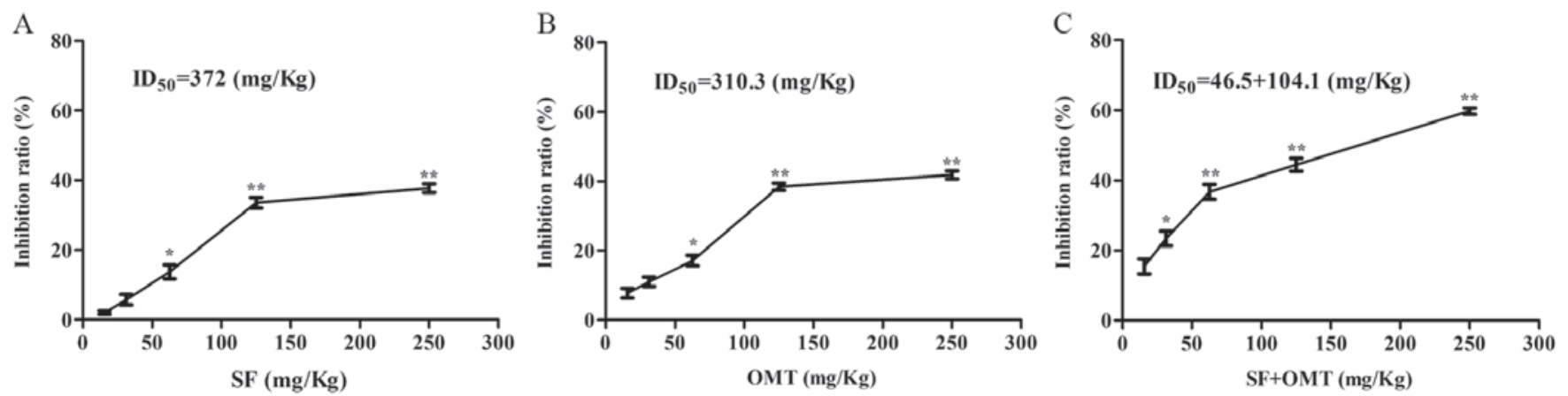

Figure 1. Inhibitory effects of SF and OMT administrated alone or in combination on exudation in mice with acetic acid-induced peritonitis as detected via staining of the peritoneal lavage fluid following perfusion with Evans blue solution. Values are expressed as the mean \pm standard error of the mean ( $\mathrm{n}=10$ ). The inhibition ratio compared with the peritonitis model group was detected in (A) the SF and (B) the OMT at concentrations of $15.6,31.3,62.5,125 \mathrm{and} 250 \mathrm{mg} / \mathrm{kg}$ and (C) the SF + OMT group at different concentrations of SF and OMT [4.8+10.8 (15.6), 9.7+21.6 (31.3), 19.4+43.1 (62.5), 38.8+86.2 (125) and 77.5+172.5 (250) $\mathrm{mg} / \mathrm{kg}$. ${ }^{*} \mathrm{P}<0.05,{ }^{* *} \mathrm{P}<0.01$, compared with the model group. SF, sodium ferulate; OMT, oxymatrine; $\mathrm{ID}_{50}$, dose at which $50 \%$ inhibition was achieved.

area, which indicated that the two drugs exerted a synergistic anti-exudation effect (Fig. 2).

Effects of sodium ferulate and oxymatrine administered alone or in combination on the number of leukocytes in the peritoneal lavage fluid. The effects of sodium ferulate and oxymatrine administered alone or in combination on the leukocyte number in the peritoneal lavage fluid were determined in the mouse model of acetic acid-induced peritonitis (Fig. 3). Treatment with sodium ferulate and oxymatrine combination $(9.7+21.6,19.4+43.1$ and $38.8+86.2 \mathrm{mg} / \mathrm{kg})$ significantly decreased the leukocyte number in the peritoneal lavage fluid compared with that in the model group $(\mathrm{P}<0.05$ in the low-dose group; $\mathrm{P}<0.01$ in the medium- and high-dose group). The inhibition ratio was up to $72.6 \%$ in the high-dose group. In addition, the results demonstrated that the leukocyte number in the peritoneal lavage fluid was also decreased in the oxymatrine monotreatment group $(\mathrm{P}<0.05)$, but not in the sodium ferulate monotreatment group at a dose equivalent to the medium dose in the combination treatment (19.4 and $43.1 \mathrm{mg} / \mathrm{kg}$, respectively). In the DEX group, the leukocyte number in the peritoneal lavage fluid significantly decreased compared with that in the model group $(\mathrm{P}<0.01$; Fig. 3), but no statistical significance was observed when the DEX group was compared with the combination drug treated group.

Effects of sodium ferulate and oxymatrine administered alone or in combination on the levels of IL-6, CRP and IFN- $\gamma$ in the peritoneal lavage fluid. As shown in Fig. 4, the levels of IL-6, $\mathrm{CRP}$ and IFN- $\gamma$ in the peritoneal lavage fluid were significantly increased in the model group compared with those in the control group. Compared with the model group, after treatment with sodium ferulate and oxymatrine combination, the levels of IL-6, CRP and IFN- $\gamma$ in the peritoneal lavage fluid were significantly decreased in a dose-dependent manner, and the effect was better than that in the monotreatment groups. In DEX group, the levels of IL-6, CRP and IFN- $\gamma$ in the peritoneal lavage fluid also significantly decreased compared with that in the model group ( $\mathrm{P}<0.01$; Fig. 4$)$, and no statistical significance was observed when compared with the combination drug treated group.

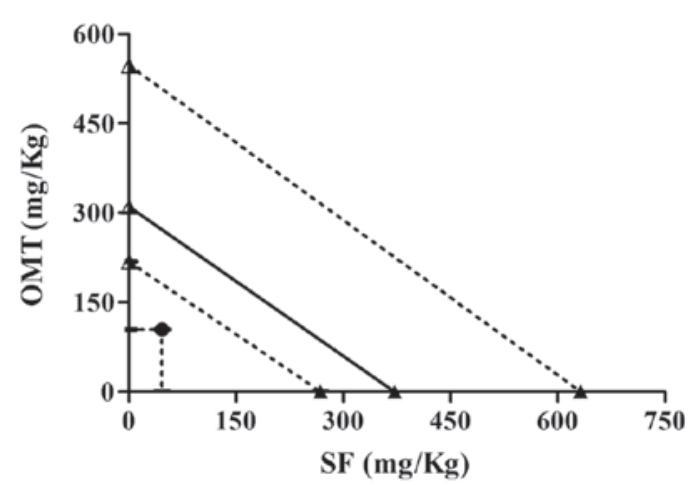

Figure 2. Isobologram demonstrating the synergistic effect of SF and OMT combination on the exudation inhibition ratio of Evans blue. The ID $_{50}$ values and $95 \%$ confidence limits of SF and OMT were marked on the x-axis and $\mathrm{y}$-axis, respectively. The $\mathrm{ID}_{50}$ values were linked with a solid line and the $95 \%$ confidence limits were linked with dashed lines. The experimental ID $_{50}$ (solid dot) of the combination was $46.5 \mathrm{mg} / \mathrm{kg} \mathrm{SF}+104.1 \mathrm{mg} / \mathrm{kg} \mathrm{OMT}$, and it was under the isobol of the theoretical additive area, indicating a synergistic interaction between the two drugs. SF, sodium ferulate; OMT, oxymatrine; $\mathrm{ID}_{50}$, dose at which $50 \%$ inhibition was achieved.

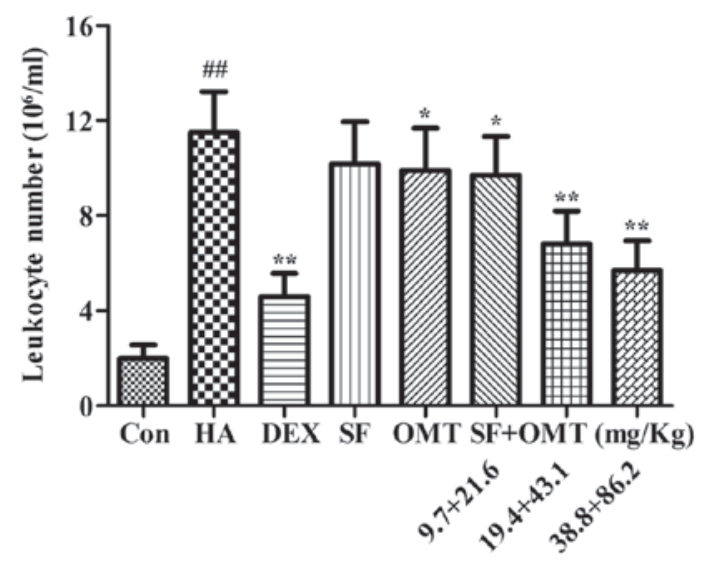

Figure 3. Effects of SF and OMT administered alone or in combination on the number of leukocytes in the peritoneal lavage fluid of mice with acetic acid-induced peritonitis. Groups: Con, control group; HA, model group (saline and acetic acid); DEX, model mice treated with DEX; SF, model mice treated with SF $(19.4 \mathrm{mg} / \mathrm{kg})$; OMT, model mice treated with OMT $(43.1 \mathrm{mg} / \mathrm{kg}) ; \mathrm{SF}+\mathrm{OMT}$, model mice treated with SF and OMT at the indicated doses. Values are expressed as the mean \pm standard error of the mean $(\mathrm{n}=10) .{ }^{\# \#} \mathrm{P}<0.01$, vs. the control group. ${ }^{*} \mathrm{P}<0.05,{ }^{* *} \mathrm{P}<0.01$, compared with the model group. DEX, dexamethasone; SF, sodium ferulate; OMT, oxymatrine. 

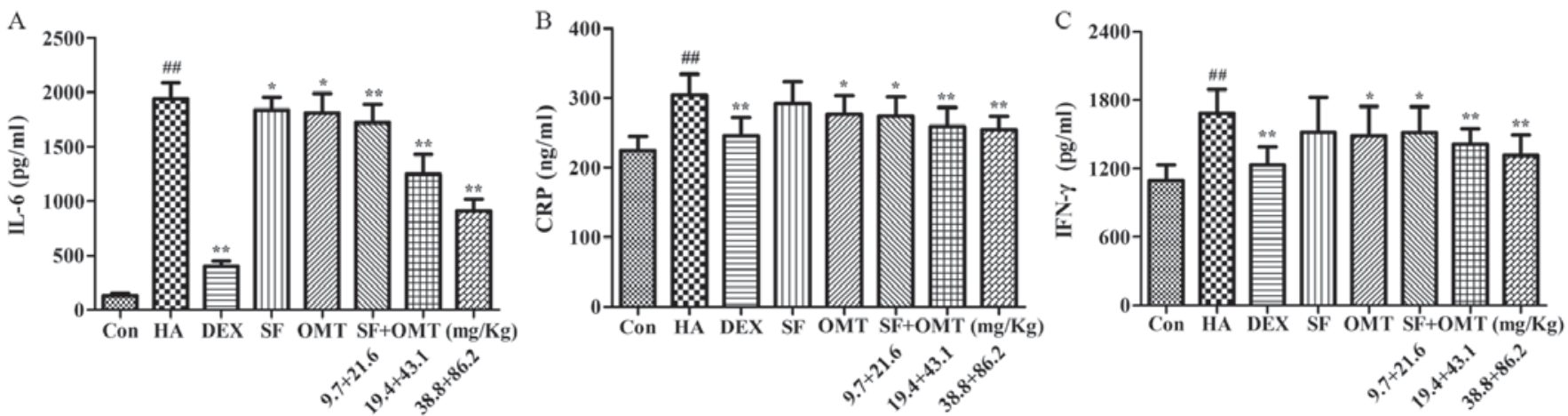

Figure 4. Effects of sodium ferulate and oxymatrine administered alone or in combination on the levels of (A) IL-6, (B) CRP and (C) IFN- $\gamma$ in the peritoneal lavage fluid. Groups: Con, control group; HA, model group (saline and acetic acid); DEX, model mice treated with DEX; SF, model mice treated with SF $(19.4 \mathrm{mg} / \mathrm{kg}) ;$ OMT, model mice treated with OMT $(43.1 \mathrm{mg} / \mathrm{kg}) ; \mathrm{SF}+\mathrm{OMT}$, model mice treated with SF and OMT at the indicated doses. Values are expressed as the mean \pm standard error of the mean $(\mathrm{n}=10) .{ }^{\sharp \#} \mathrm{P}<0.01$, vs. the control group. ${ }^{*} \mathrm{P}<0.05,{ }^{* *} \mathrm{P}<0.01$, compared with the model group. DEX, dexamethasone; $\mathrm{SF}$, sodium ferulate; OMT, oxymatrine; IL, interleukin; CRP, C-reactive protein; IFN, interferon.
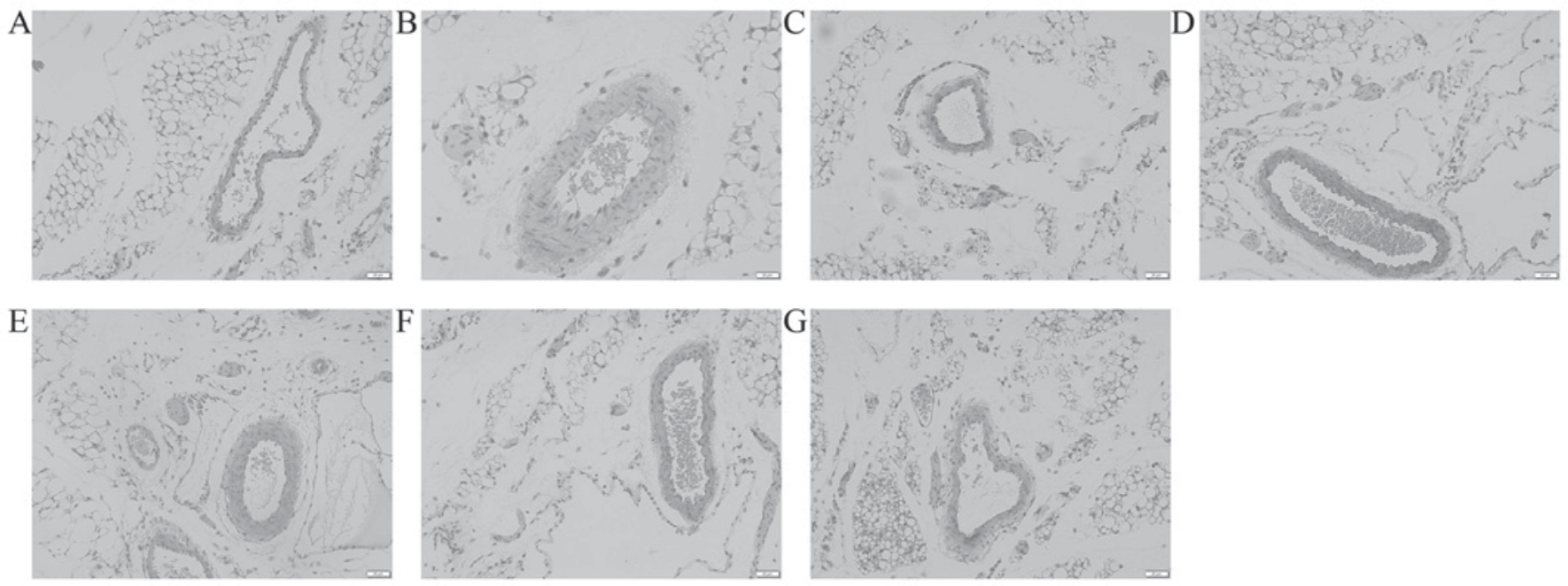

Figure 5. Pathological examination of omentum majus of the mice by hematoxylin and eosin staining. (A) Control group; (B) model group (saline and acetic acid); (C) sodium ferulate (19.4 mg/kg); (D) oxymatrine ( $43.1 \mathrm{mg} / \mathrm{kg}$ ); (E) sodium ferulate and oxymatrine combination $(9.7+21.6 \mathrm{mg} / \mathrm{kg}) ;(\mathrm{F}) \mathrm{sodium}$ ferulate and oxymatrine combination $(19.4+43.1 \mathrm{mg} / \mathrm{kg}) ;(\mathrm{G})$ sodium ferulate and oxymatrine combination $(38.8+86.2 \mathrm{mg} / \mathrm{kg})$. Magnification, x200.

Effects of sodium ferulate and oxymatrine administered alone or in combination on vascular endothelial cell morphology, capillary construction and the expression of AQP1 in omentum majus. Pathological analysis of omentum majus revealed that compared to the control group, the vascular endothelial cell morphology and capillary construction were severely damaged in the model group, including increased inter-cell gaps, protrusion into the lumen or exfoliation leading to capillary loss (Fig. 5A and B). After treatment with sodium ferulate and oxymatrine combination, the damage was significantly alleviated in a dose-dependent manner. The damage was also alleviated in the oxymatrine monotreatment group, but the effect was less than that of sodium ferulate and oxymatrine combined (Fig. 5C-G). The omentum majus damage score were significantly and dose-dependently improved in the sodium ferulate and oxymatrine combination treatment groups, and a significant improvement was also seen in the oxymatrine monotreatment group (Fig. 6).

The expression of AQP1 in vascular endothelial cells of the omentum majus was evaluated by immunohistochemistry
(Fig. 7). The results showed that the OD of AQP1 in the model group was significantly decreased compared with that in the control group. However, the OD was significantly higher in the sodium ferulate and oxymatrine combination groups compared with that in the model group $(\mathrm{P}<0.05$ in the low-dose combination group; $\mathrm{P}<0.01$ in the medium- and high-dose group). Sodium ferulate or oxymatrine monotreatment group did not significantly increase the OD of AQP1.

Effects of sodium ferulate and oxymatrine administered alone or in combination on the cell volume and the AQP1 expression of HUVEC. As shown in Table I, the forward scatter integral area (FSC-A) and side scatter integral area (SSC-A) were significantly increased in the model group compared with those in the control group. However, in the combination groups $(50+100,100+200$ or $200+400 \mu \mathrm{mol} / \mathrm{l})$, the FSC-A and SSC-A were significantly reduced in a dose-dependent manner. The expression of AQP1 was detected by immunocytochemisty (Fig. 8). The results showed that the OD of AQP1 was significantly decreased in the model group, compared with that in 
Table I. Effects of sodium ferulate and oxymatrine administrated alone or in combination on the volume of human umbilical vein endothelial cells.

\begin{tabular}{|c|c|c|c|c|c|}
\hline \multirow[b]{2}{*}{ Group } & \multirow[b]{2}{*}{$\begin{array}{c}\text { Dose of } \mathrm{SF}+ \\
\mathrm{OMT}(\mu \mathrm{mol} / \mathrm{l})\end{array}$} & \multicolumn{2}{|c|}{ FSC-A } & \multicolumn{2}{|c|}{ SSC-A } \\
\hline & & Value & $\begin{array}{l}\text { Increase } \\
\text { (\% of Con) }\end{array}$ & Value & $\begin{array}{l}\text { Increase } \\
\text { (\% of Con) }\end{array}$ \\
\hline Con & - & $116,811 \pm 5493$ & - & $67,967 \pm 4047$ & - \\
\hline HA & - & $137,640 \pm 8045$ & $17.84 \pm 0.04^{\mathrm{a}}$ & $96,798 \pm 9664$ & $42.67 \pm 0.15^{\mathrm{a}}$ \\
\hline $\mathrm{SF}$ & $100+0$ & $130,728 \pm 1086$ & $11.99 \pm 0.09$ & $87,912 \pm 8113$ & $29.58 \pm 0.13$ \\
\hline OMT & $0+200$ & $130,060 \pm 5632$ & $11.397 \pm 0.03^{b}$ & $87,404 \pm 6818$ & $28.87 \pm 0.11^{b}$ \\
\hline $\mathrm{SF}+\mathrm{OMT}$ & $50+100$ & $127,716 \pm 4853$ & $9.55 \pm 0.07^{b}$ & $82,389 \pm 9034$ & $21.39 \pm 0.13^{\mathrm{b}}$ \\
\hline $\mathrm{SF}+\mathrm{OMT}$ & $100+200$ & $122,910 \pm 4883$ & $5.47 \pm 0.08^{c}$ & $76,866 \pm 5579$ & $13.23 \pm 0.08^{c}$ \\
\hline $\mathrm{SF}+\mathrm{OMT}$ & $200+400$ & $122,213 \pm 6612$ & $4.6 \pm 0.02^{c}$ & $74,389 \pm 3167$ & $9.65 \pm 0.06^{c}$ \\
\hline
\end{tabular}

Values are expressed as the mean \pm standard error $(\mathrm{n}=4)$. ${ }^{\mathrm{a}} \mathrm{P}<0.01$, compared with the control group; ${ }^{\mathrm{b}} \mathrm{P}<0.05,{ }^{\mathrm{c}} \mathrm{P}<0.01$, compared with the model group. Con, control; HA, model group (saline and acetic acid); SF, sodium ferulate; OMT, oxymatrine; FSC-A, forward scatter area; SSC-A, side scatter area.

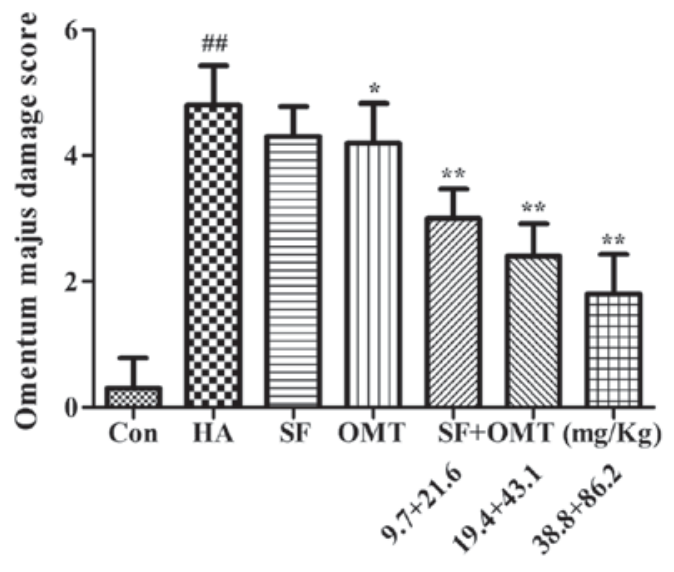

Figure 6. Effects of SF and OMT administered alone or in combination on pathological changes of omentum majus in a mouse model of acetic acid-induced peritonitis. The omentum majus damage score is shown. Groups: Con, control group; HA, model group (saline and acetic acid); SF, model mice treated with $\mathrm{SF}(19.4 \mathrm{mg} / \mathrm{kg})$; OMT, model mice treated with OMT $(43.1 \mathrm{mg} / \mathrm{kg}) ; \mathrm{SF}+\mathrm{OMT}$, model mice treated with SF and OMT at the indicated doses. Values are expressed as the mean \pm standard error of the mean $(\mathrm{n}=10)$. ${ }^{\# \#} \mathrm{P}<0.01$, vs. the control group. ${ }^{*} \mathrm{P}<0.05,{ }^{* *} \mathrm{P}<0.01$, compared with the model group. SF, sodium ferulate; OMT, oxymatrine.

the control group. However, compared with the model group, the OD in the sodium ferulate and oxymatrine combination treatment groups was significantly increased when the concentration was $>50+100 \mu \mathrm{mol} / 1$. Furthermore, the OD of AQP1 in the sodium ferulate or oxymatrine monotreatment groups was significantly elevated compared with that in the model group when the drug concentration was $>100 \mu \mathrm{mol} / 1(\mathrm{P}<0.05)$.

\section{Discussion}

The inflammatory response, a basic pathological process, is characterized by cell damage, inflammatory exudation and tissue hyperplasia, including cellular edema, degeneration and necrosis, tissue fibrosis as well as inflow of blood components and inflammatory corpuscles to damaged tissue (21). Among

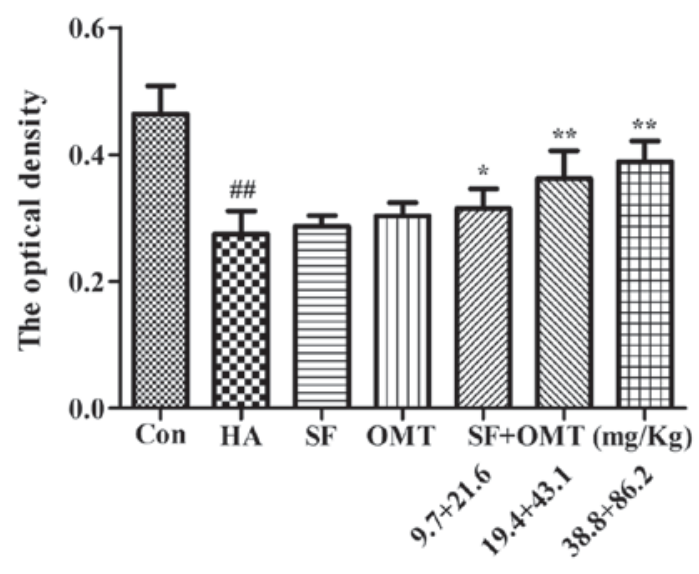

Figure 7. Effects of SF and OMT administered alone or in combination on the expression of AQP1 in vascular endothelial cells of the omentum majus. The optical density was used to represent the strength of expression of AQP1. Groups: Con, control group; HA, model group (saline and acetic acid); SF, model mice treated with SF $(19.4 \mathrm{mg} / \mathrm{kg})$; OMT, model mice treated with OMT $(43.1 \mathrm{mg} / \mathrm{kg}) ; \mathrm{SF}+\mathrm{OMT}$, model mice treated with SF and OMT at the indicated doses. Values are expressed as the mean \pm standard error of the mean $(\mathrm{n}=10) .{ }^{\# / \prime} \mathrm{P}<0.01$, vs. the control group. ${ }^{*} \mathrm{P}<0.05,{ }^{* *} \mathrm{P}<0.01$, compared with the model group. SF, sodium ferulate; OMT, oxymatrine; AQP, aquaporin.

them, inflammatory exudation and cellular edema are major pathophysiological characteristics in the early phase of the inflammatory response meaning acute inflammation, and usually lead to vulnerability to organ damage such as acute lung edema and peritonitis (22). Previous studies by our group found that sodium ferulate and oxymatrine combination significantly alleviate the acute inflammatory response (23). Based on a comprehensive analysis of these results, it was hypothesized that the drug may have a significant anti-exudation effect. Among the numerous animal models, the mouse model of acetic acid-induced peritonitis is commonly used to estimate anti-exudation effects of drugs (12), and is characterized by increased vascular permeability, fluid exudation, leukocyte migration and inflammatory cytokine release to the abdominal cavity (24). In this model, the degree of 

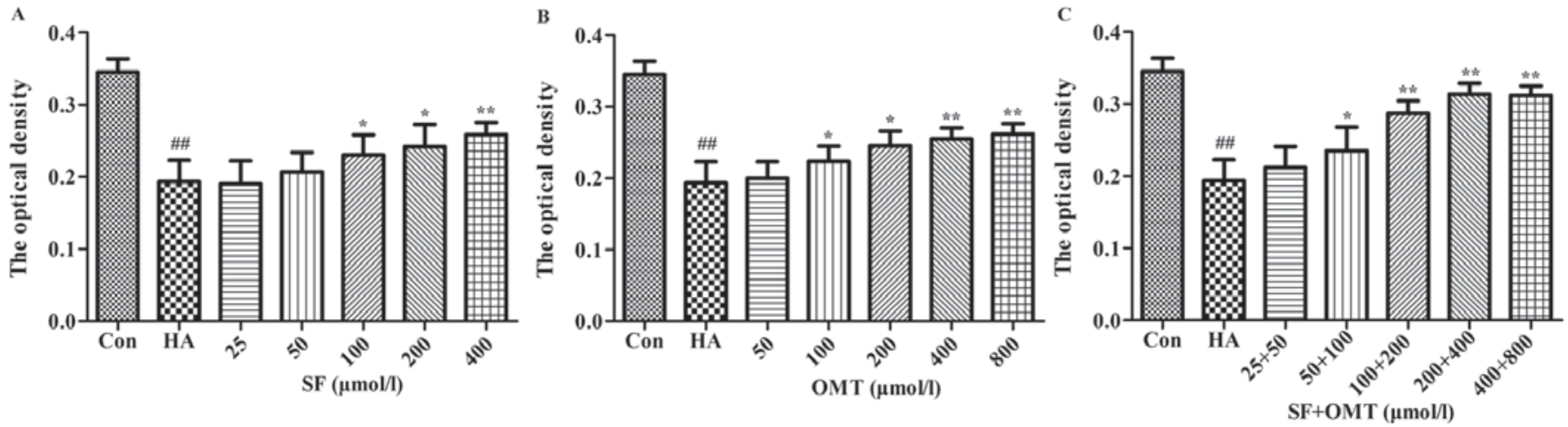

Figure 8. Effects of (A) SF (B) OMT and (C) SF + OMT administered alone or in combination on the expression of AQP1 in human umbilical vein endothelial cells. The optical density was used to represent the strength of expression of AQP1. Values are expressed as the mean \pm standard error of the mean $(\mathrm{n}=6)$. ${ }^{\# \#} \mathrm{P}<0.01$, vs. the control group. ${ }^{\prime} \mathrm{P}<0.05,{ }^{* *} \mathrm{P}<0.01$, compared with the model group. $\mathrm{SF}$, sodium ferulate; OMT, oxymatrine; Con, control group; HA, model group (saline and acetic acid).

inflammatory exudation is evaluated by non-subjective quantification of these indicators.

Evans blue is a chemical dye, which easily combines with plasma protein. Only when capillary permeability is increased, Evans blue combined with protein inflows to the abdominal cavity from capillaries. Following intravenous injection of Evans blue and induction of peritonitis with acetic acid in mice, the amount of Evans blue leakage reflecting vascular permeability may be evaluated by measurement of its OD value at $590 \mathrm{~nm}$ in the peritoneal lavage fluid and the inhibition ratio was calculated as previously described (25). In the present study, the OD value of Evans blue in the peritoneal lavage fluid was markedly increased in the model group, meaning that plasma protein leaked out of blood vessels and reflecting that the vascular permeability was increased. Treatment with sodium feulate and oxymatrine combination significantly relieved the acetic acid-induced inflammatory exudation in a dose-dependent manner, as represented by an increased exudation inhibition ratio of Evans blue, and its effect was better than that of the each drug alone. More importantly, the isobologram analysis demonstrated that the experimental $\mathrm{ID}_{50}$ of sodium ferulate and oxymatrine combination was under the isobol of the theoretical additive area, indicating that the two drugs possessed a synergistic anti-exudation effect.

With increasing vascular permeability and leukocyte migration to the inflammatory site, large amounts of inflammatory cytokines were released, which were found in the model group. Treatment with sodium feulate and oxymatrine combination also significantly decreased the leukocyte number as well as the levels of IL-6, CRP and IFN- $\gamma$ in the peritoneal lavage fluid induced by acetic acid, and its effect was better than that of each drug alone at doses equal to those in the medium combined group, which verified the results of previous studies by our group, which reported that sodium ferulate and oxymatrine combination significantly reduced the levels of IL-6, CRP and IFN- $\gamma$ in serum or tissue homogenate of other mouse models $(5,6,23)$. IL-6, a pro-inflammatory cytokine, has a vital role in the process of inflammation and macrophage activation (26-28). CRP is a non-specific biomarker of inflammation, which is closely associated with tissue injury (29). IFN- $\gamma$ is also a potent pro-inflammatory cytokine resulting in damage of tissue (30). Comprehensive analysis of the anti-inflammatory effects of certain drugs revealed that they reduced the permeability of blood vessels, inhibited inflammatory corpuscle inflow to the inflammatory site and reduced the release of inflammatory factors, which indicated that these effects may be a cascading reaction.

A recent study found that damaged vascular endothelial cells are among the reasons for increased vascular permeability (31). In the present study, the vascular endothelial cellular morphology and blood capillary morphology were assessed. The vascular endothelial cellular morphology and the integrity of blood capillaries were significantly reduced in the model group. However, treatment with sodium ferulate and oxymatrine combination significantly improved the surface morphology of vascular endothelial cells and alleviated the amount of damaged blood capillaries. In order to study the effect of the drugs on vascular endothelial cell edema, volume changes of HUVECs were assessed by flow cytometry. Flow cytometry is a novel technology using a laser beam to measure single cells in the flow to rapidly and accurately analyze or quantitate their physical and chemical characteristics. FSC is a parameter measuring light scattered by $<10^{\circ}$ as a cell passes through the laser beam to detect the surface properties of cells. The FSC value is proportional to cell size (32), and its signal is expressed as FSC-A. Treatment with sodium ferulate and oxymatrine combination significantly reduced the FSC-A value, indicating that drugs in combination alleviate cellular edema. This suggested that the anti-exudation effect of the combination of sodium ferulate and oxymatrine may be associated with alleviating vascular endothelial cellular edema. In addition, SSC, also referred to as $90^{\circ}$ scatter or right-angle scatter, includes light scattered at a $90^{\circ}$ angle as a cell passes through the laser beam and reflects its interior properties. The SSC value is associated with the internal granularity or complexity of a particle, and its signal is expressed as the SSC-A. After treatment with the combination of sodium ferulate and oxymatrine, the SSC-A value was also decreased, indicating a decrease of intracellular particles, which will be further elucidated in a future study.

It is well-known that increased intracellular water is the basic characteristic of cellular edema (33). AQP1 is a water channel protein and its function is mainly to transport water across the plasma membrane, contributing to water 
homeostasis of cells (34). AQP1 is expressed in a great majority of microvascular endothelia, as well as in endothelial cells of tissues including the cornea and intestinal lacteals (35). Kim et al (36) found that decreased expression of AQP1 in the choroid plexus led to a decrease in cerebrospinal fluid formation. The present study therefore hypothesized that vascular endothelial cellular edema induced by acetic acid was associated with increased AQP1 expression, and after the drug treatments, the expression of AQP1 decreased in vascular endothelial cells, which reduced vascular endothelial cellular edema, alleviated capillary permeability and decreased the amount of peritoneal fluid induced by acetic acid. To verify this surmise, the effects of sodium ferulate and oxymatrine combination on AQP1 expression on the membrane of the vascular endothelial cells of the omentum majus and HUVECs were assessed. However, immunohistochemical analysis of omentum majus and HUVECs revealed that the expression of AQP1 was significantly decreased after acetic acid stimulation, while it was significantly increased after treament with a combination of sodium ferulate and oxymatrine, which is the opposite effect of what was expected. The possible explanation is that the vascular endothelial cellular edema induced by acetic acid were due to water retention through an increase in intracellular metabolism, which was caused by a reduction of transportation channels represented by their decreased expression. After the drug treatments, the expression of AQP1 increased in vascular endothelial cells, which was in parallel with a reduction of vascular endothelial cellular edema and alleviation of the increased capillary permeability induced by acetic acid. Furthermore, in the present study, intracellular particulate substance was significantly increased after acetic acid stimulation, which was represented by an elevated SSC-A in the flow cytometric analyses, and may be one of the major causes of cellular edema. The type of intracellular particulates that are increased and the underlying mechanism, as well as the mechanism of intracellular water accumulation will be assessed in future studies. For answering these questions, appropriate methods are to be identified.

In conclusion, sodium ferulate and oxymatrine combination exhibited significant and synergistic anti-exudation effects, alleviating vascular endothelial cellular edema induced by acetic acid. The significant effects of the drugs on AQP1 and the underlying mechanisms require further exploration.

\section{References}

1. Lee JH, Shin H, Kim YJ, Paek SH, Jin S and Ha UH: Pseudomonas aeruginosa-induced IL- $1 \beta$ production is inhibited by Sophora flavescens via the NF- $\mathrm{KB}$ /inflammasome pathways. J Microbiol 52: 1044-1049, 2014.

2. Xiao TT, Wang YY, Zhang Y, Bai CH and Shen XC: Similar to spironolactone, oxymatrine is protective in aldosterone-induced cardiomyocyte injury via inhibition of calpain and apoptosis-inducing factor signaling. PLoS One 9: e88856, 2014.

3. Wang W, Pei X, Xu M, Sun S, Zhang C, Mu K and Liu Z: The protective effect of sodium ferulate and oxymatrine combination on paraquat-induced lung injury. Iran J Pharm Res 14 573-583, 2015

4. Yuan X, Sun Y, Miao N, Sun S, Wang Y, Hu Z, Yuan J, Xu M and Liu Z: The synergistic anti-inflammatory effect of the combination of sodium ferulate and oxymatrine and its modulation on inflammation-associated mediators in RAW 264.7 cells. J Ethnopharmacol 137: 1477-1485, 2011.
5. Xu M, Wang W, Pei X, Sun S, Xu M and Liu Z: Protective effects of the combination of sodium ferulate and oxymatrine on cecal ligation and puncture-induced sepsis in mice. Exp Ther Med 7: 1297-1304, 2014.

6. Pei X, Wang W, Miao N, Xu M, Zhang C, Sun M, Xu M and Liu Z: The protective effects of the combination of sodium ferulate and oxymatrine on ethanol-induced liver damage in mice. Environ Toxicol Pharmacol 37: 423-430, 2014.

7. Da Cruz RB, Galdino PM, Penna KG, Hoffmann K, Costa EA and Bataus LA: Acetone extract from Streptoverticillium sp., a bacterium isolated from Brazilian Cerrado soil, induces anti-inflammatory activity in mice. An Acad Bras Cienc 85: 595-603, 2013.

8. Syam S, Bustamam A, Abdullah R, Sukari MA, Hashim NM, Mohan S, Looi CY, Wong WF, Yahayu MA and Abdelwahab SI: $\beta$ Mangostin suppress LPS-induced inflammatory response in RAW 264.7 macrophages in vitro and carrageenan-induced peritonitis in vivo. J Ethnopharmacol 153: 435-445, 2014.

9. Zhao C, Zhang Y, Zou P, Wang J, He W, Shi D, Li H, Liang G and Yang S: Synthesis and biological evaluation of a novel class of curcumin analogs as anti-inflammatory agents for prevention and treatment of sepsis in mouse model. Drug Des Devel Ther 9: $1663-1678,2015$

10. Gao J, Tan M, Gu M, Marshall C, Ding J, Hu G and Xiao M: Cellular localization of aquaporin-1 in the human and mouse trigeminal systems. PLoS One 7: e46379, 2012.

11. National Institutes of Health (US): Using Animals In Intramural Research: Guidelines for Investigators and Guidelines for Animal Users. NIH Animal Care and Use Committee, 1994.

12. Barros WM, Rao VS, Silva RM, Lima JC and Martins DT: Anti-inflammatory effect of the ethanolic extract from Bowdichia virgilioides H.B.K stem bark. An Acad Bras Cienc 82: 609-616, 2010.

13. Shen Q, Wu MH and Yuan SY: Endothelial contractile cytoskeleton and microvascular permeability. Cell Health Cytoskelet 2009: 43-50, 2009.

14. Di Franco M, Paradiso M, Riccieri V, Basili S, Mammarella A and Valesini G: Autonomic dysfunction and microvascular damage in systemic sclerosis. Clin Rheumatol 26: 1278-1283, 2007.

15. Hou S, Zheng F, Li Y, Gao L and Zhang J: The protective effect of glycyrrhizic acid on renal tubular epithelial cell injury induced by high glucose. Int J Mol Sci 15: 15026-15043, 2014.

16. Mu H, Lin KX, Zhao H, Xing S, Li C, Liu F, Lu HZ, Zhang Z, Sun YL, Yan XY, et al: Identification of biomarkers for hepatocellular carcinoma by semiquantitative immunocytochemistry. World J Gastroenterol 20: 5826-5838, 2014.

17. Park MS, Kim CS, Joo HK, Lee YR, Kang G, Kim SJ, Choi S, Lee SD, Park JB and Jeon BH: Cytoplasmic localization and redox cysteine residue of APE1/Ref-1 are associated with its anti-inflammatory activity in cultured endothelial cells. Mol Cells 36: 439-445, 2013.

18. Jang J, Kim HS, Kang JW and Kang HC: The genetically modified polysialylated form of neural cell adhesion molecule-positive cells for potential treatment of X-linked adrenoleukodystrophy. Yonsei Med J 54: 246-252, 2013.

19. Calero C, López-Campos JL, Izquierdo LG, Sánchez-Silva R, López-Villalobos JL, Sáenz-Coronilla FJ, Arellano-Orden E, Montes-Worboys A and Echevarría M: Expression of aquaporins in bronchial tissue and lung parenchyma of patients with chronic obstructive pulmonary disease. Multidiscip Respir Med 9: 29, 2014.

20. Li SB, Yang KS and Zhang YT: Expression of aquaporins 1 and 3 in degenerative tissue of the lumbar intervertebral disc. Genet Mol Res 13: 8225-8233, 2014.

21. Toffoli-Kadri MC, Carollo CA, Lourenço LD, Felipe JL, Néspoli JH, Wollf LG, Resende GM, de Lima JR, Franco VN, Vieira Mdo $\mathrm{C}$ and de Siqueira JM: In vivo and in vitro anti-inflammatory properties of Achyrocline alata (Kunth) DC. J Ethnopharmacol 153: 461-468, 2014.

22. Mizuno M, Ito Y, Mizuno T, Harris CL, Suzuki Y, Okada N, Matsuo S and Morgan BP: Membrane complement regulators protect against fibrin exudation increases in a severe peritoneal inflammation model in rats. Am J Physiol Renal Physiol 302: F1245-F1251, 2012.

23. Yuan X, Wang Y, Du D, Hu Z, Xu M, Xu M and Liu Z: The effects of the combination of sodium ferulate and oxymatrine on lipopolysaccharide-induced acute lung injury in mice. Inflammation 35: 1161-1168, 2012. 
24. Smeding L, Plötz FB, Lamberts RR, van der Laarse WJ, Kneyber MC and Groeneveld AB: Mechanical ventilation with high tidal volumes attenuates myocardial dysfunction by decreasing cardiac edema in a rat model of LPS-induced peritonitis. Respir Res 13: 23, 2012.

25. Yu HL, Zhang F, Li YJ, Gong GH and Quan ZS: Anti-inflammatory and antinociceptive effects of 6-(4-chlo rophenoxy)-tetrazolo[5,1-a]phthalazine in mice. Pharmacol Rep 64: 1155-1165, 2012.

26. Hsing $\mathrm{CH}$ and Wang JJ: Clinical implication of perioperative inflammatory cytokine alteration. Acta Anaesthesiol Taiwan 53: 23-28, 2015.

27. Niu X, Yao H, Li W, Mu Q, Li H, Hu H, Li Y and Huang $\mathrm{H}$ : $\delta$-Amyrone, a specific inhibitor of cyclooxygenase-2, exhibits anti-inflammatory effects in vitro and in vivo of mice. Int Immunopharmacol 21: 112-118, 2014

28. Arteaga Figueroa L, Barbosa Navarro L, Patiño Vera M and Petricevich VL: Preliminary studies of the immunomodulator effect of the Bougainvillea xbuttiana extract in a mouse model. Evid Based Complement Alternat Med 2015: 479412, 2015.

29. de Oliveira TH, Amorin AT, Rezende IS, Santos Barbosa M, Martins HB, Brito AK, Andrade EF, Gonçalves GK Campos GB, Silva RA, et al: Sepsis induced by Staphylococcus aureus: Participation of biomarkers in a murine model. Med Sci Monit 21: 345-355, 2015.
30. de Lima TH, Sass N, Mattar R, Moron AF, Torloni MR, Franchim CS and Daher S: Cytokine gene polymorphisms in preeclampsia and eclampsia. Hypertens Res 32: 565-569, 2009.

31. Zhao J, Chen L, Shu B, Tang J, Zhang L, Xie J, Liu X, Xu Y and Qi S: Granulocyte/macrophage colony-stimulating factor attenuates endothelial hyperpermeability after thermal injury. Am J Transl Res 7: 474-488, 2015.

32. Jordan CT, Yamasaki G and Minamoto D: High-resolution cell cycle analysis of defined phenotypic subsets within primitive human hematopoietic cell populations. Exp Hematol 24: 1347-1355, 1996.

33. Ma X, Shatil-Cohen A, Ben-Dor S, Wigoda N, Perera IY, Im YJ, Diminshtein S, Yu L, Boss WF, Moshelion M and Moran N: Do phosphoinositides regulate membrane water permeability of tobacco protoplasts by enhancing the aquaporin pathway? Planta 241: 741-755, 2015.

34. Moon C, Preston GM, Griffin CA, Jabs EW and Agre P: The human aquaporin-CHIP gene. Structure, organization, and chromosomal localization. J Biol Chem 268: 15772-15778, 1993.

35. Verkman AS: Aquaporin water channels and endothelial cell function. J Anat 200: 617-627, 2002.

36. Kim JG, Son YJ, Yun CH, Kim YI, Nam-Goong IS, Park JH, Park SK, Ojeda SR, D'Elia AV, Damante G and Lee BJ: Thyroid transcription factor-1 facilitates cerebrospinal fluid formation by regulating aquaporin-1 synthesis in the brain. J Biol Chem 282: 14923-14931, 2007. 